\title{
PRELIMINARY REPORT ON THE USE OF CHLORAMPHENICOL (CHLOROMYCETIN) IN THE TREATMENT OF ACUTE GONORRHEAL URETHRITIS ${ }^{1}$
}

\author{
By J. E. SMADEL, C. A. BAILEY, AND D. S. MANKIKAR \\ (From the Army Medical Department Research and Graduate School, the Commission on \\ Immunization of the Army Epidemiological Board, Washington, D. C., and the \\ Institute for Medical Research, Kuala Lumpur, Malaya)
}

The great advances which have been made in recent years in the treatment of gonorrheal infections would appear to have provided the medical profession with adequate therapeutic agents for this disease. The numerous advantages of penicillin treatment have resulted in its replacing the sulfonamide drugs, except in selected cases (1). Even the disadvantages of parenteral injections of penicillin have been avoided by a number of workers who have administered the antibiotic by mouth (1). Indeed, Campbell, Dougherty and Curtis reported on the oral use of penicillin in the prophylaxis of gonorrhea at an earlier session of this Symposium (2). Despite the fact that penicillin has many attributes of an ideal drug for the treatment of gonorrhea, it has one distinct drawback, i.e., its suppressive effect on a coexisting syphilitic infection. This problem of the possible masking of syphilis by treating acute gonorrhea patients with amounts of penicillin which are adequate for the bacterial infection but inadequate for the spirochetal infection is of tremendous importance $(1,3)$. Therefore, the search must continue for a drug with the activity of penicillin against the gonococcus but without its marked anti-spirochetal action. This report is a progress note which presents certain preliminary data suggesting that chloramphenicol, or Chloromycetin, ${ }^{2}$ approaches penicillin in its efficacy in the treatment of acute gonorrheal urethritis in the male and that this new drug is distinctly less spirocheticidal than penicillin.

1 Presented at the Second National Symposium on Recent Advances in Antibiotics Research held in Washington, D. C., April 11-12, 1949, under the auspices of the Antibiotics Study Section, National Institutes of Health, Public Health Service, Federal Security Agency.

2 Trade name of Parke, Davis and Company. The Chloromycetin used in the present work was supplied by Parke, Davis and Company. Lots of drug produced by the fermentation and by the synthetic process were made available.
During the past three months in Malaya we have used Chloromycetin in treating 45 men with gonorrhea. Thirty-four of these received the synthetic type of drug and 11 the fermentation type. Data on 32 patients in this series are summarized in Table I. Each person had a purulent urethral discharge when admitted to the study. This had been present for three days or less in the majority of instances, but three individuals in the group had suffered for 21,28 , and 60 days, respectively. The mean duration of disease when therapy was begun was from four to 12 days in the four subgroups listed in Table I. Smears of the exudate taken from each patient

TABLE I

Chloromycetin in the treatment of 32 males with acute gonorrheal urethritis

\begin{tabular}{|c|c|c|c|c|}
\hline & \multicolumn{4}{|c|}{ Chloromycetin dosage } \\
\hline & \multicolumn{2}{|c|}{ Synthetic } & \multicolumn{2}{|c|}{ Fermentation } \\
\hline & $\begin{array}{c}3.0-3.5 \\
\text { grams }\end{array}$ & $\underset{\text { grams }}{1.0-1.5}$ & $\begin{array}{c}3.0-3.5 \\
\text { grams }\end{array}$ & $\begin{array}{c}1.0-1.5 \\
\text { grams }\end{array}$ \\
\hline $\begin{array}{l}\text { Number of cases } \\
\text { Day of illness treatment } \\
\text { begun } \\
\text { Mean } \\
\text { Range }\end{array}$ & $\begin{array}{c}11 \\
12 \\
1-60 z\end{array}$ & $\begin{array}{c}10 \\
4 \\
1-10\end{array}$ & $\stackrel{8}{1-21}$ & $\begin{array}{c}4 \\
1-10\end{array}$ \\
\hline $\begin{array}{l}\text { Urethral discharge } \\
\text { Hours duration post- } \\
\text { treatment } \\
\text { Mean } \\
\text { Range }\end{array}$ & $\begin{array}{c}43 \\
5-72\end{array}$ & $\begin{array}{c}43 \\
24-72\end{array}$ & $\begin{array}{c}53 \\
7-96\end{array}$ & $\begin{array}{c}42 \\
18-60\end{array}$ \\
\hline
\end{tabular}

on admission contained numerous Gram-negative intracellular diplococci. Furthermore, cultures, made on Harrison's agar, yielded $N$. gonococci from all but one of the cases; the single exception suffered a relapse on the eighth day and is included because of this.

Each of the patients was treated with a single oral dose of one or the other type of Chloromycetin and permitted to continue his normal occupational duty. Slightly more than half the patients listed in the table received a total of 3.0 or 3.5 grams and the remainder were given 1.0 or 1.5 grams. It is apparent from the tabular data that similar results were obtained as regards disappearance of urethral discharge in the four subgroups, i.e., patients who received the smaller or larger dose of either the synthetic or fermentation type of drug. Later on 
additional comments will be made on other patients not mentioned in the table who were treated with the smaller doses of drug.

Within 24 hours after the therapeutic dose had been given the urethral discharge had disappeared in 13 of the 32 patients. Furthermore, in another 17 of the group the discharge was reduced to a drop or so of thin watery material which was obtained by stripping. Two of the 32 persons still yielded a drop of purulent exudate at this time. Stained smears of the 19 exudates revealed that only one had Gram-negative intracellular diplococci; the culture of this material failed to yield $N$. gonococci. However, a few colonies of $N$. gonococci were grown from a thin milky exudate of another patient who was cultured 24 hours after being given 3.0 grams of drug. Furthermore, the material from three other patients taken at this time yielded colonies resembling $N$. gonococci.

On the third to fifth days after treatment was given, 30 of the 32 patients were subjected to prostatic massage. Materials for smear and culture were obtained from 18 of these. In no instance were Gram-negative intracellular diplococci found microscopically ; indeed, even leukocytes were recorded as being absent from the smears of 12 of the specimens. Seventeen of the 18 cultures of prostatic secretion taken during this period contained no $N$. gonococci but from one secretion a few colonies were grown which resembled gonococci morphologically. Three subsequent massages of Patient 4, who had supplied this questionable material, were non-productive and he has now remained well for eight weeks.

A total of four relapses have been noted to date among the 32 patients who have been tabulated. One occurred 28 days after treatment with 3.0 grams of synthetic drug, which was 26 days after complete disappearance of all signs of disease. This individual had been re-exposed recently and he may represent a reinfection. Two patients relapsed on the 19th and eighth days, respectively, after treatment with 3.0 grams of synthetic drug; discharges of both had initially cleared within 48 hours and recurred following inebriation. The fourth case was apparently cured within 36 hours after receiving 1.0 gram of fermentation drug but relapsed on the 14th day. Each of these four patients was again treated with a single dose of 3.0 grams of Chloromycetin and responded well.
Three additional patients not included in the table are worthy of mention. Each received a single dose of 1.0 or 1.5 grams of Chloromycetin but was subsequently given an additional 3.0gram dose one to four days later. This additional medication appeared indicated because of the persistence of some discharge, or the presence of Gram-negative intracellular diplococci. However, none of the cultures taken at the time the supplementary drug was administered were later found to contain gonococci. Each of the three patients recovered promptly after being given the additional drug. Thus, it appears that the administration of 1.0 gram or 1.5 grams of drug in a single dose may provide inadequate therapy in a proportion of cases.

Smith and his coworkers (4) found that rabbits which had been infected with the Nichols strain of Treponema pallidum and then treated with Chloromycetin for eight days at the rate of $25 \mathrm{mg}$. per kilo per day showed no change in their lesions nor did the spirochetes disappear. They also noted that daily doses of 50 and $100 \mathrm{mg}$. per kilo cleared the lesions of spirochetes but that the effect was temporary.

Our experience with Chloromycetin in two patients with primary chancres suggests that the results in man may be similar to those just mentioned in rabbits. Dark field examination of fluid from the lesion of one man showed an average of three motile spirochetes per field. The patient received 4.0 grams of Chloromycetin in a single dose (45 mg. per kilo of body weight) and repeated examinations over the next 18 hours revealed the same number of active treponema. He was then given 3.0 grams of drug at one time followed by five doses of 0.5 gram each at three-hour intervals, thus totaling 5.5 grams (62 mg. per kilo) over this 18-hour period. At the end of this time two spirochetes of reduced motility were found in the three preparations examined and eight hours later only one non-motile organism was seen in two samples. During the succeeding week the chancre healed slowly and spirochetes were not observed. The second patient was given an initial dose of $70 \mathrm{mg}$. per kilo of body weight and an additional $58 \mathrm{mg}$. per kilo over the next 15 hours. Seventeen hours after treatment was started motile spirochetes could no longer be found but there was 
no evidence of healing of the chancre during the succeeding three days.

\section{CONCLUSIONS}

The present information, although preliminary, indicates that Chloromycetin is of some value in the treatment of acute gonorrheal urethritis in the male. Single oral doses have been employed almost exclusively in these studies; the efficacy of the same total amounts of drug given in divided doses over a period of one or two days should be investigated.

Chloromycetin is appreciably less effective than penicillin against Treponema pallidum. Since an agent capable of controlling gonorrhea without masking syphilitic infection is to be desired, further studies of the use of Chloromycetin in the treatment of venereal disease are indicated.

\section{ADDENDUM}

Since the above was submitted additional cases have been added to this series and supplementary data obtained on the original patients. One lot of chloramphenicol, presumed to be fermentation type, later proved to be synthetic; this necessitated minor changes in the tabulation of the data. A total of 48 patients with acute gonorrheal urethritis have been treated with chloramphenicol. Of these, 42 were given synthetic drug in a single oral dose. Twenty-four were given 3.0 to 3.5 grams and 18 were given 1.0 to 1.5 grams. The remaining six received 3.0 to 3.5 grams of fermentation drug. The mean duration of disease in these three subgroups prior to treatment was 8, 4, and 11 days, respectively. The clinical response was essentially the same in each sub-group; therefore, neither the type of drug nor the dosage employed appeared to affect the results. Dysuria disappeared within 36 hours (average for the 48 patients) and discharge (as determined by the absence of exudate on stripping) within 49 hours. Smears of the discharge with few exceptions were free of gonococci by the next day and leukocytes generally were absent from the discharge in those instances where this sign persisted longer than 48 hours. Ten patients or 20 per cent of this entire series had relapses during the month following treatment. There was no apparent difference in the number of recur- rences in the three sub-groups. All relapses were treated with a single oral 3.0 gram dose of chloramphenicol and responded satisfactorily.

The primary lesion of the first patient with a syphilitic chancre mentioned in the text, who received 9.5 grams of chloramphenicol over a twoday period, healed completely by the tenth day after beginning treatment. During the fourth week a chancre containing spirochetes reappeared at the site of the original lesion. The patient was then retreated, receiving three single 1.0 gram oral doses of synthetic drug at daily intervals. Spirochetes disappeared from the recurrent chancre the day following the third dose of drug and the lesion was completely healed nine days later. On the 44th day after the first appearance of the chancre and the 36th day after initial treatment, a Kahn test gave a plus-minus reaction; it had been consistently negative previously. The three chancres of the second patient, who received 5.5 grams during 18 hours, healed by the 12 th day but 30 days later a fresh chancre reappeared at one of the original sites. The Kahn reaction of the second patient remained negative throughout the 35 days of observation following the original appearance of the lesions.

Nine patients with acute gonorrheal urethritis were treated with single oral doses of 3.0 to 4.25 grams of aureomycin. In this small series the clinical effect was essentially similar to that obtained with chloramphenicol and the results were somewhat better than those obtained by Collins et al. (5). This may be due to our use of larger amounts of drug. The mean duration of disease before treatment in this series was 15 days. Dysuria and discharge disappeared on the average in 29 and 82 hours, respectively. In all cases smears were negative for gonococci by the day after treatment. These patients were followed for an average of 28 days during which time there were no relapses.

\section{BIBLIOGRAPHY}

1a. Kolmer, J. A., Penicillin Therapy Including Streptomycin, Tyrothricin and Other Antibiotic Therapy. D. Appleton-Century Company, Inc., New York, 1947, Ed. 2, pp. 188-194.

b. Dowling, H. F., The Acute Bacterial Diseases, Their 
Diagnosis and Treatment. W. B. Saunders Company, Philadelphia, 1948, pp. 230-232.

2. Campbell, V. W. H., Dougherty, W. J., and Curtis, C. E., Delayed administration of oral penicillin as prophylaxis for gonorrhea. J. Clin. Invest., 1949, 28, 1040.

3. Walker, A. E., and Barton, R. L., The treatment of gonorrhea with penicillin during the incubation period or early phase of syphilis-a review. J. Vener. Dis. Information, 1945, 26, 241.

4. Smith, R. M., Joslyn, D. A., Gruhzit, O. M., McLean, I. W., Jr., Penner, M. A., and Ehrlich, J., Chloromycetin : biological studies. J. Bact., 1948, 55, 425.

5. Collins, H. S., Paine, T. F., Jr., and Finland, M., Clinical studies with aureomycin. Annals of the N. Y. Acad. of Sciences, 1948, 51, 231. 